\title{
Headache as presenting symptom of neurosarcoidosis
}

\author{
M. Curone $\cdot$ V. Tullo $\cdot$ C. Peccarisi • \\ G. Bussone $\cdot$ D. D'Amico
}

(C) Springer-Verlag Italia 2013

\begin{abstract}
Headache may occur in neurosarcoidosis and diagnostic criteria are given in the International Classification of Headache Disorders (ICHD-II). We present a case series of patients suffering from neurosarcoidosis in whom headache was the presenting symptom. The aim of the present study was to analyze the possible clinical presentations and the corresponding cerebral lesions in a retrospective chart review of patients suffering from neurosarcoidosis in whom headache was the presenting symptom. Medical records and data of six patients were analyzed. The possible diagnoses of headache forms included in ICDH-II, and in particular the correspondence with the criteria for "headache attributed to neurosarcoidosis", as well as neuroimaging findings were evaluated in each patient. The ICHD-II criteria were fulfilled in all the six patients. As for as clinical presentation, in three patients $(50 \%)$, headache had the clinical characteristics of TolosaHunt syndrome, with evidence of a lesion into the cavernous sinus. In the remaining three cases headache was the only neurological symptom found in association with systemic features of sarcoidosis, and had the clinical features of tension-type headache. Our findings confirm that the clinical features of headache in patients with this disorder may have different presentations, which depend on neuropathologic involvement. Thus, a detailed neuroimaging study and CSF evaluation are needed to confirm diagnosis, particularly in patients with no sign of systemic sarcoidosis
\end{abstract}

M. Curone $(\bowtie) \cdot$ V. Tullo · C. Peccarisi · G. Bussone .

D. D'Amico

Headache Unit, Department of Clinical Neurological Sciences,

Neurological Institute C. Besta, IRCCS Foundation,

Via Celoria 11, 20133 Milan, Italy

e-mail: marcella.msc.doc@gmail.com or in those in whom head pain may mimic a primary headache syndrome.

Keywords Neurosarcoidosis - Sarcoidosis · Headache · Inflammatory diseases

\section{Introduction}

Sarcoidosis is a multisystemic disease of unknown origin, characterized by non-caseating granulomas in affected organs. Neurological complications in sarcoidosis are rare, highly variable. The appropriate diagnostic investigations include magnetic resonance imaging (MRI) of the brain and spinal cord, cerebrospinal fluid studies, brain and meningeal biopsy if feasible, chest radiography, angiotensin-converting enzyme level in the serum or in the cerebrospinal fluid (CSF) [1, 2]. Kveim test [2] and fluorodeoxyglucose positron emission tomography/computed tomography (FDG-PET) $[3,4]$ can be performed in particular cases.

Neurological involvement may present as cranial neuropathy (facial palsy, optic nerve or other cranial nerve involvement), peripheral neuropathy, and manifestations of the central nervous system affecting hypothalamus, pituitary gland, cerebral cortex, cerebellum, meninges, and spinal cord [1]. Neurosarcoidosis accounts for approximately $5 \%$ of cases of sarcoidosis [5]. Among neurological symptoms, headache may occur [1], and diagnostic criteria of "headache attributed to neurosarcoidosis" are given by International Headache Society (IHS) in the 2004 headache classification (ICHD-II) in chapter 7 under "headaches attributed to non-vascular intracranial disorders" [6] (Table 1). Different kinds of headache are likely to be observed, and headache characteristics depend on the 
Table 1 "Headache attributed to neurosarcoidosis": diagnostic criteria of the International Headache Society (ICHD-II)

A. Headache, no typical characteristics known, fulfilling criteria C and D

B. Evidence of neurosarcoidosis ${ }^{\mathrm{a}}$

C. Headache develops in temporal relation to neurosarcoidosis

D. Headache resolves within 3 months after successful treatment of neurosarcoidosis

${ }^{a}$ Evidence of neurosarcoidosis includes cranial nerve lesions, intracranial space-occupying lesion on MRI, aseptic meningitis and/or periventricular inflammatory focal lesions and homogeneously enhancing mass lesions that are confirmed on biopsy as non-caseating granulomas

nature of the cerebral involvement and vary within and across the underlying lesions. Intractable occipital pain radiating frontally with nausea and visual disturbances has been reported in patients with isolated supratentorial tumour-like lesions [7]. Diffuse or bifrontal pain is a more typical symptom of leptomeningeal involvement, associated or not to papilledema $[8,9]$. Other forms of cranial pain may be related to trigeminal or optic nerve involvement [10, 11]; migraine [12] and painful ophthalmoplegia [13] have been also reported.

The aim of the present study was to analyze the possible clinical presentations and the corresponding cerebral lesions in a retrospective chart review of patients suffering from neurosarcoidosis in whom headache was the presenting symptom.

\section{Methods}

The charts of patients admitted to our Headache Unit in the past 10 years for headache evaluation with a final diagnosis of neurosarcoidosis were retrospectively reviewed. The diagnosis of neurosarcoidosis and systemic sarcoidosis was performed according to brain or spinal magnetic resonance imaging, thoracic CT scan, and/or gallium scintigraphy, bronchoscopy, cerebrospinal fluid study, increased angiotensin-converting enzyme presence and response to steroid treatment.

Medical records, data regarding clinical features, headache characteristics and MRI findings were analyzed. The possible diagnoses of headache forms included in ICDH-II, and in particular the correspondence with the criteria for "headache attributed to neurosarcoidosis" was evaluated in each patient.

\section{Results}

Six patients, four males, two females, mean age at observation 40.3 years (range 29-56) were included in the review; headache was the onset symptom in all. In four patients $(67 \%)$ headache had acute onset; in the remaining two

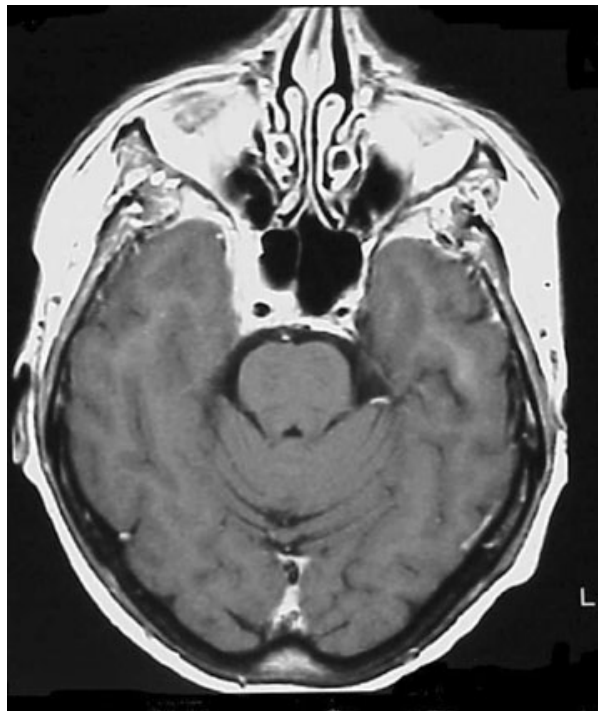

Fig. 1 Axial T1-weighted MRI image showing inflammatory process in the right cavernous sinus in a patient with painful ophthalmoplegia due to neurosarcoidosis

patients headache had chronic course and was reported as mild at the beginning, but subsequently worsening. Signs of systemic disease were present in four patients. Intracranial lesions were observed at MRI in all the headache patients.

As far as clinical presentation, in three patients (50\%), the headache was unilateral, periorbital, intense and sharp associated with oculomotor palsy (two with complete third nerve palsy and one with six nerve palsy and transient ipsilateral trigeminal neuralgia). A lesion into the ipsilateral cavernous sinus was found in these three patients reporting painful ophthalmoplegia, consisting in abnormal soft tissue isointense to grey matter with enhancement after contrast medium administration (Fig. 1). In these patients, a diagnosis of Tolosa-Hunt syndrome could be made, but steroid treatment (prednisone administrated orally at $60 \mathrm{mg} /$ day) allowed pain remission within 1 week, i.e. with a latency which was longer than the $72 \mathrm{~h}$ latency required by diagnostic criteria of this syndrome, according to ICHD-II [6]. In the remaining three cases (50\%) headache was the only neurological symptom found in association with systemic features of sarcoidosis. Pain was 
diffuse, severe, non-pulsating, with daily or nearly-daily occurrence, without migrainous associated symptoms and no-response to steroid treatment. In these patients the clinical presentation mimicked chronic tension-type pain, but increased meningeal enhancement around the brainstem was detected in two of them, and several white matter inflammatory focal lesions were observed in the other one. The IHS criteria were fulfilled in all the six patients.

\section{Conclusions}

Headache is not a common symptom of neurosarcoidosis.

Our findings from a retrospective neurosarcoidosis series confirm that the clinical features of headache in patients with this disorder may have different presentation [2, 4, 13]. Our cases were clinically similar to Tolosa-Hunt syndrome or to tension-type headache.

Our survey highlights some aspects which can be useful in clinical practice: headache attributed to neurosarcoidosis has no typical characteristics, as stated in the ICDH-II; different kinds of headache may occur in relation to neuropathologic involvement; a detailed neuroimaging study and CSF evaluation are needed in patients presenting with headache and suffering from systemic sarcoidosis or in whom isolated neurosarcoidosis is suspected.

Therefore, a diagnosis of "headache attributed to neurosarcoidosis" remains very challenging, also because neurosarcoidosis is a low prevalent disease. This is particularly true in patients without evidence of systemic sarcoidosis or in those in whom head pain may mimic a primary headache syndrome, with a high risk of delaying a correct diagnosis.

Conflict of interest I certify that there is no actual or potential conflict of interest in relation to this article.

\section{References}

1. Tsao CY, Lo WD, Rusin JA, Henwood MJ, Boue (2007) Isolated neurosarcoidosis presenting as headache and multiple brain and spinal cord lesions mimicking central nervous system metastases. Brain Dev 29(8):514-518

2. Nozaki K, Judson MA (2012) Neurosarcoidosis: clinical manifestations, diagnosis and treatment. Presse Med 41(6):331-348

3. Bartels S, Kyavar L, Blumstein N, Görg T, Glöckner SC, Zimmermann R, Heckmann JG (2013) FDG PET findings leading to diagnosis of neurosarcoidosis. Clin Neurol Neurosurg 115(1):85-88

4. Kim SK, Im HJ, Kim W, Kim TS, Hwangbo B, Kim HJ (2010) F-18 fluorodeoxyglucose and F-18 fluorothymidine positron emission tomography/computed tomography imaging in a case of neurosarcoidosis. Clin Nucl Med 35(2):67-70

5. Gascón-Bayarri J, Mañá J, Martínez-Yélamos S, Murillo O, Reñé R, Rubio F (2011) Neurosarcoidosis: report of 30 cases and a literature survey. Eur J Intern Med 22(6):125-132

6. Headache Classification Subcommittee of the International Headache Society (2004) The international classification of headache disorders: 2nd edition. Cephalalgia 24(Suppl 1):9-16

7. Vannemreddy PSSV, Nanda A, Reddy PK, Gonzalez E (2002) Primary cerebral sarcoid granuloma: the importance of definite diagnosis in the high-risk patient population. Clin Neurol Neurosurg 104:289-292

8. Katz JM, Bruno MK, Winterkorn JMS, Nealon N (2003) The pathogenesis and treatment of optic disk swelling in neurosarcoidosis. Arch Neurol 60:426-430

9. Zouaoui A, Maillard JC, Dormont D, Chiras J, Marsalult C (1992) MRI in neurosarcoidosis. J Neuroradiol 19:271-284

10. Braido F, Zolezzi A, Stea F, Canonica GW, Perotti L, Cavallero GB, Genovese E, Gozzoli L (2005) (2005) Bilateral Gasser's ganglion sarcoidosis: diagnosis, treatment and unsolved questions. Sarcoidosis Vasc Diffuse Lung Dis 22(1):75-77

11. Amin A, Balderacchi JL (2010) Trigeminal neurosarcoidosis: case report and literature review. Ear Nose Throat J 89(7):320-2 (review)

12. Dizdarevic K, Disdarevic S, Disdarevic Z (1998) Neurosarcoidosis presenting with transitory neurodeficit and generalized epileptic seizures associated with migraine. Med Arh 52:159-162

13. Curone M, Tullo V, Proietti-Cecchini A, Peccarisi C, Leone M, Bussone G (2009) Painful ophthalmoplegia: a retrospective study of 23 cases. Neurol Sci 30 (suppl 1):S133-S135 